Bol. Acad. peru. leng. 63. 2018 (129-153)

\title{
EL PRINCIPIO DE CONCORDANTIA TEMPORUM EN LAS CLÁUSULAS NOMINALES DEL CASTELLANO PERUANO
}

\section{THE CONCORDANTIA TEMPORUM RULE IN PERUVIAN SPANISH NOUN CLAUSES}

\author{
Claudia Crespo del Río \\ Pontificia Universidad Católica del Perú (PUCP)
}

\section{Resumen:}

El principio de Concordantia o Consecutio Temporum señala que el tiempo del verbo subordinado en subjuntivo concuerda con el tiempo del verbo principal (Quiero que duerma / Quería que durmiera), pero es posible hallar que no se cumple (Quería que duerma) en muchas variedades. En el caso del castellano peruano, este estudio busca ver si este fenómeno es frecuente en bilingües en Ayacucho y monolingües en Lima con mayor o menor influencia migrante. Estos grupos son comparados con uno mexicano para comprobar que el cambio es más frecuente en el Perú. Además, se incluyen factores lingüísticos (el tipo de verbo principal y el tipo de evento) para confirmar si el no cumplimiento del principio ocurre incluso en los contextos más estrictos. Los resultados y estadísticas confirman que no se cumple en el castellano peruano en todos los contextos y en mayor frecuencia que en el castellano mexicano. Asimismo, las comparaciones entre grupos señalan que 
los grupos limeños son similares, lo que muestra el intenso contacto social, y que los bilingües tienden a producir otros modos gramaticales, como el indicativo. Un análisis más detallado sugiere que esas respuestas son preferidas por los hablantes con un menor nivel de dominio de castellano, ya que el subjuntivo se adquiere tardíamente al desarrollar una segunda lengua.

\section{Abstract:}

The Concordantia or Consecutio Temporum rule states that the verb tense in a noun clause agrees with the verb tense in the main clause (Quiero que duerma "I want [present indicative] her to sleep [present subjunctive]" / Quería que durmiera "I wanted [imperfect indicative] her to sleep [imperfect subjunctive]"), but it is possible to find many varieties that do not follow the rule (Quería que duerma "I wanted [imperfect indicative] her to sleep [present subjunctive]"). In Peruvian Spanish, this study aims to find whether this phenomenon is frequent in bilinguals in Ayacucho and in monolinguals in Lima with more migrant contact or less migrant contact. These groups are compared to a Mexican group in order to confirm if the change is more common in Peru. Moreover, linguistic factors (verb type in the main clause and event type) are included to see if the rule is not followed even within the strictest contexts. Results and statistical analyses show that this is true and more frequent than in Mexican Spanish. Furthermore, between-group comparisons point out that groups in Lima are similar, which supports the intense social contact in the capital. In addition to that, bilinguals tend to produce other grammatical moods, as indicative. A more detailed analysis suggests that these answers are preferred by speakers that are less dominant in Spanish, since subjunctive is acquired in later stages of the second language acquisition process.

Palabras clave: Concordantia Temporum; subjuntivo; español peruano; contacto lingüístico; bilingüismo.

Key words: Concordantia Temporum; subjunctive; Peruvian Spanish; linguistic contact; bilingualism.

Fecha de recepción: $\quad$ 14/03/2018

Fecha de aceptación: 31/05/2018 
https://doi.org/10.46744/bapl.201801.005

\section{Introducción}

Los diversos estudios sobre el español peruano muestran que existen diferencias resaltantes entre variedades geográficas y sociales, las que pueden ser explicadas a partir de diversos criterios, como características socioeconómicas, lugares de procedencia, situaciones de bilingüismo, etc. (Caravedo 1992, Pérez Silva 2004). Si bien se reconocen tales diferencias, también es relevante considerar que muchos fenómenos gramaticales y otros elementos lingüísticos se han extendido en el país debido al constante contacto social entre hablantes de diferentes procedencias (Caravedo y Klee 2005, Escobar 2000).

Así, este estudio se enfoca en tres variedades peruanas y en su producción de formas del subjuntivo en cláusulas subordinadas nominales. En particular, buscamos describir qué tan frecuente es el principio de Concordantia o Consecutio Temporum (CT), por el que el tiempo del verbo subordinado debe seguir al tiempo del verbo principal. Si se trata de un fenómeno más cercano a la norma estándar, nos interesa enfocarnos en lo que sucede en variedades que son comúnmente estigmatizadas o calificadas de manera negativa. Al comparar tres variedades, se pretende corroborar si esos hablantes comparten el mismo patrón de producción del principio o se diferencian por sus características sociolingüísticas.

Una investigación como esta contribuye a ampliar el panorama de estudios sobre el castellano peruano, sobre todo con respecto al contacto lingüístico entre variedades dialectales y sociales, puesto que se trata de un fenómeno cercanamente ligado al intenso contacto social que existe en el Perú. Además, los resultados ampliarán aún más la información acerca de cómo el principio de CT continúa perdiendo vigencia entre los dialectos latinoamericanos de español.

El artículo está organizado de la siguiente manera: la primera sección se ocupa de describir el principio de CT y cómo es usado en el español peruano y latinoamericano. Luego, la siguiente caracteriza el castellano peruano y la situación de contacto sociolingüístico que explica las 
coincidencias entre variedades; así, sobre la base de la información vertida en ambas secciones, se plantean las tres hipótesis del estudio antes de pasar a la tercera sección. Esta última detalla los aspectos metodológicos de la investigación: los participantes, los factores lingüísticos y sociales, y el instrumento diseñado. A continuación, se presentan los resultados y se ofrece una discusión de estos, de modo que se puedan verificar o refutar las hipótesis planteadas.

\section{Estudios sobre Concordantia Temporum}

En español, el principio de Concordantia Temporum o Consecutio Temporum (Gili Gaya 1948) ha sido planteado como la secuencia temporal que siguen el verbo de la cláusula principal y el de la cláusula subordinada. Así, en las cláusulas subordinadas nominales, los verbos en subjuntivo siguen el tiempo de los verbos de la cláusula principal, como lo muestran (1) y (2).

(1) Quiero que duerma.

(2) Quería que durmiera.

El ejemplo (1) muestra que ambos verbos comparten el tiempo presente, mientras que en el ejemplo (2) comparten el tiempo pasado. En la normativa de español, se espera que esta concordancia se cumpla. Por otro lado, en cuanto a variedades dialectales, algunas se acercan más que otras a este principio. En aquellas que no siguen el principio, se generan cambios en las oraciones cuyo verbo principal está en pasado. Así, se producen ejemplos como (3).

\section{(3) Quería que duerma.}

Tal como se observa, el ejemplo (3) muestra que el verbo de la cláusula principal está en pasado (pretérito imperfecto), pero el verbo de la cláusula subordinada no sigue la secuencia, pues está en presente subjuntivo. Este fenómeno de cambio ha sido descrito en variedades habladas en la región andina y en otras zonas del Perú (Sessarego 2008, 2010, Arrizabalaga 2009). 
Suñer y Padilla-Rivera (1987) se ocupan del principio de CT y proponen que es posible encontrar variación entre pasado y presente subjuntivo según los requisitos semánticos y pragmáticos del verbo en la cláusula principal. Así, se establecen seis tipos de verbos para la cláusula principal, tal como se muestra en la Tabla 1.

Tipos de verbos en la cláusula principal, tomado de Suñer y Padilla-Rivera (1987) ${ }^{1}$

\begin{tabular}{|c|c|c|c|}
\hline $\begin{array}{l}\text { Tipo de verbo } \\
\text { en la cláusula } \\
\text { principal }\end{array}$ & $\begin{array}{l}\text { Tiempo en } \\
\text { la cláusula } \\
\text { principal }\end{array}$ & $\begin{array}{l}\text { Tiempo en } \\
\text { la cláusula } \\
\text { subordinada }\end{array}$ & $\begin{array}{l}\text { Combinaciones de } \\
\text { tiempo no aceptadas }\end{array}$ \\
\hline Negación & {$[ \pm$ pasado $]$} & {$[ \pm$ pasado $]$} & Ninguna \\
\hline $\begin{array}{l}\text { Factivo- } \\
\text { emotivo }\end{array}$ & [土pasado $]$ & [土pasado $]$ & Ninguna \\
\hline \multirow{2}{*}{ Incertidumbre } & [-pasado] & [土pasado] & \multirow{2}{*}{$\begin{array}{l}{[+ \text { pasado }] \ldots} \\
{[\text {-pasado }]}\end{array}$} \\
\hline & [+ pasado $]$ & {$[+$ pasado $]$} & \\
\hline \multirow{2}{*}{ Influencia } & [-pasado] & [-pasado] & \multirow{2}{*}{$\begin{array}{l}{[\text {-pasado }] \ldots} \\
{[+ \text { pasado }]}\end{array}$} \\
\hline & {$[+$ pasado $]$} & [+pasado] & \\
\hline \multirow{2}{*}{ Deseo } & [-pasado] & {$[ \pm$ pasado $]$} & \multirow{2}{*}{$\begin{array}{l}{[+ \text { pasado }] \ldots} \\
{[\text {-pasado }]}\end{array}$} \\
\hline & [+ pasado $]$ & {$[+$ pasado $]$} & \\
\hline $\begin{array}{l}\text { Falta de } \\
\text { conocimiento }\end{array}$ & {$[+$ pasado $]$} & {$[+$ pasado $]$} & $\begin{array}{l}{[+ \text { pasado }] \ldots} \\
{[\text {-pasado }]}\end{array}$ \\
\hline
\end{tabular}

Tabla 1

El primer y el segundo tipo son los más flexibles con respecto al cumplimiento del principio. Esto supone que es normativamente aceptable encontrar presente subjuntivo con verbos como negar, o alegrarse o lamentar, como lo muestra el ejemplo (4).

1 La traducción es mía. 
(4) Me alegré de que ella siga estudiando.

Por el contrario, los últimos dos tipos de verbos son los más estrictos en exigir que se cumpla con CT. Así, verbos como querer, desear o ignorar aceptan solo una forma de pasado subjuntivo en la cláusula subordinada, como se ve en (5a). Sin embargo, tal como se indicó antes, esto no ocurre en todas las variedades: en las peruanas, más bien, sí se acepta el presente subjuntivo, como en (5b).

(5a) Quería que telefonearas.

(5b) Quería que telefonees.

Además de este criterio, se debe considerar el momento del evento de habla o tiempo de la comunicación, como los autores mencionados lo denominan (TOC o time of communication). Este segundo factor es relevante al interpretar las oraciones, pues la exigencia de cumplir con el principio de CT puede pasarse por alto en el caso de que el evento de la cláusula subordinada sea posterior al tiempo de la comunicación. Así, por ejemplo, si tomamos (5b), podemos entender que el evento de telefonear aún no se ha realizado y es posible de realizar en el momento en que se produce la oración.

De este modo, es interesante observar qué ocurre en el castellano peruano, pues, si se trata de variedades que no siguen el principio, es posible encontrar casos de presente subjuntivo incluso cuando el evento ya es imposible de realizar en el tiempo de la comunicación. Este caso extremo ha sido estudiado por otros autores, como Sessarego (2008); su estudio se enfoca en esos casos en que la normativa exige un verbo en pasado. Él analiza 865 cláusulas subordinadas del español boliviano y del español peruano, las cuales forman parte de textos escritos que integran el Corpus de Referencia del Español Actual (CREA). Estos textos corresponden a artículos periodísticos y libros escritos por periodistas y escritores que representan la norma educada de cada país. Asimismo, las diferencias dialectales pueden ser identificadas solamente a nivel de país, ya que ese es el único dato de origen que se consigna en el CREA para cada autor. 
https://doi.org/10.46744/bapl.201801.005

El autor considera el factor del género del texto (periódicos o libros), que muestra que $40 \%$ del presente subjuntivo se halla en artículos periodísticos y solo $3 \%$ en libros, lo que da una diferencia estadísticamente significativa. En cuanto al dialecto, el español boliviano tiene una mayor tendencia a no cumplir CT que el peruano (37\% versus $9 \%$ ).

Dentro del castellano peruano, se tomaron en cuenta diversos factores, pero solo la clase del verbo y la agentividad resultaron estadísticamente significativos: casi el $50 \%$ de respuestas tienen presente subjuntivo cuando hay un verbo de creación en la cláusula principal; estos verbos expresan creencias, deseos o suposiciones. Con respecto a agentividad, $65 \%$ de los sujetos no-agentes favorecieron el incumplimiento de CT.

De este modo, el autor concluye que el castellano peruano aún mantiene parámetros fuertes, a diferencia de lo que ocurre en el boliviano. No obstante, se trata de resultados que no especifican las variedades regionales o sociales de los hablantes, y que hacen evidente el manejo de la norma culta por parte de los autores.

Posteriormente, en 2010, Sessarego realiza un nuevo estudio que toma en cuenta veinte dialectos latinoamericanos. Sin embargo, no todos cuentan con una cantidad similar de ocurrencias de presente subjuntivo (algunos con más de 200 ocurrencias y otros con menos de 40). Por ello, el autor aclara que se trata de conclusiones preliminares, además de que los datos también son tomados del CREA. Los resultados señalan que los países con mayor tendencia al incumplimiento de CT son Bolivia, Paraguay, Ecuador, Uruguay y Perú. Por esa razón, si queremos ver qué tanto se aleja el castellano peruano de la norma estándar, conviene comparar los resultados con una variedad que no aparezca en los primeros lugares, como la mexicana. Es así que nuestro estudio plantea una primera hipótesis: las variedades de castellano peruano se alejan más del principio de CT que las mexicanas.

Por otro lado, con respecto a factores lingüísticos, incluimos dos: el tipo de verbo en la cláusula principal y el tipo del evento, ambos basados 
en lo que estudios previos sugieren de acuerdo con sus conclusiones. De esta forma, la segunda hipótesis plantea que el cumplimiento de CT es mayor con el tipo de verbo y el tipo de evento más estrictos.

\section{Contexto sociolingüístico peruano}

El panorama lingüístico en el Perú está fuertemente definido por la intensa situación de bilingüismo presente en el país desde tiempos coloniales. Los hablantes de lenguas originarias han adquirido el castellano como segunda lengua de una manera particular, evidenciado influencias de su lengua materna y del propio proceso de adquisición (Cerrón-Palomino 2003, Escobar 1978, Escobar 2001, 2007).

Parte de la diversidad lingüística peruana incluye también a quienes tienen el castellano como lengua materna y cuyos rasgos lingüísticos evidencian influencia de lenguas andinas (Cerrón-Palomino 2003, De Granda 2003, Escobar 1978, Escobar 2000, Zavala 1999). El español andino de estos hablantes es en realidad un grupo de variedades, muchas de ellas sociales con mayor estabilidad que una variedad adquisicional (Escobar 2000). Por esta razón, se reconocen distintos grupos de hablantes: bilingües en regiones andinas, bilingües migrantes en regiones no andinas, monolingües en regiones andinas o no andinas, entre otros. Así, resulta interesante observar qué sucede en ciudades como Lima, en la que hablantes de más de una variedad se encuentran debido a las migraciones internas y generan un contacto lingüístico permanente (Caravedo y Klee, 2005, Escobar 2009).

Como consecuencia de esta situación sociolingüística, las variedades habladas en Lima se pueden diferenciar según la percepción y la valoración que les adjudican los hablantes; por ejemplo, existen variedades consideradas prestigiosas, que también son vistas como el estándar que muchos hablantes aspiran a manejar por ser relacionado con el acceso a la educación. De otro lado, estas coexisten con variedades habladas por migrantes o por quienes tienen mayor contacto con migrantes, vistas como diferentes al estándar (Mick y Palacios, 2013). Basándonos en estas valoraciones, un principio como el CT, considerado parte de la norma- 
tiva del español, podría ser principalmente identificado con variedades prestigiosas no migrantes. Así, agregamos una nueva hipótesis a nuestro estudio: las variedades con menor contacto migrante cumplirán con el principio de CT en mayor medida que las variedades con mayor contacto migrante: grupo menos migrante $>$ grupo más migrante $>$ grupo bilingüe.

A manera de síntesis, se enumeran las hipótesis planteadas en el estudio:

I. Las variedades de castellano peruano se alejan más del principio de CT que las mexicanas.

II. El cumplimiento de CT es mayor con el tipo de verbo y el tipo de evento más estrictos.

III. Las variedades con menor contacto migrante cumplirán con el principio de CT en mayor medida que las variedades con mayor contacto migrante.

\section{Metodología}

\subsection{Participantes}

Este estudio considera cuatro grupos de participantes según el área en la que viven y la variedad lingüística que hablan. El primer y el segundo grupo están conformados por hablantes limeños que viven en la capital; el tercer grupo, por hablantes ayacuchanos que viven en la ciudad de Huamanga, y el cuarto grupo, por hablantes mexicanos residentes en Ciudad de México. Este último grupo nos permite corroborar nuestra primera hipótesis, es decir, si el cambio a presente subjuntivo es más frecuente en el Perú. Por otra parte, el primer grupo representa la variedad con menos contacto migrante y más cercana al estándar, mientras que el tercero, la variedad con mayor influencia de rasgos andinos, ya que, además, se trata de hablantes bilingües quechua-español. El segundo grupo es intermedio, en tanto que se trata de hablantes limeños con mayor contacto migrante.

Las diferencias entre los dos grupos limeños fueron establecidas a partir de un cuestionario sociolingüístico, de manera que el área de 
residencia fuera el factor determinante para identificar su contacto con migrantes. Si bien Lima es una ciudad en que la mayoría de los habitantes son migrantes de primera o de segunda generación (Arellano y Burgos 2010), vale la pena tomar en cuenta que existen áreas en las que la concentración de migrantes, como los que provienen de la región andina, es mayor. En estas últimas áreas, se espera que los rasgos de variedades andinas sean más habituales y hasta adoptados por no migrantes, un fenómeno que ya se ha demostrado en estudios anteriores (Caravedo y Klee 2005, CerrónPalomino 2003, Escobar 2009).

Por otro lado, todos los participantes comparten características sociales similares, ya que son estudiantes de nivel secundario de escuelas públicas. Esta decisión permitió asegurar que pertenezcan a un mismo estrato socioeconómico, además de compartir el mismo grupo de edad y de tener una alta probabilidad de vivir en los alrededores de la escuela (y así residir en un área de mayor o menor contacto migrante en el caso de los grupos limeños). Una síntesis de los grupos es presentada en la Tabla 2.

\section{Participantes}

\begin{tabular}{|l|l|l|l|l|}
\hline Grupo & $\begin{array}{l}\text { Ayacucho } \\
\text { bilingüe } \\
(\mathrm{n}=23)\end{array}$ & $\begin{array}{l}\text { Lima } \\
\text { Más migrante } \\
(\mathrm{n}=27)\end{array}$ & $\begin{array}{l}\text { Lima } \\
\text { Menos migrante } \\
(\mathrm{n}=24)\end{array}$ & $\begin{array}{l}\text { México } \\
(\mathrm{n}=19)\end{array}$ \\
\hline $\begin{array}{l}\text { Edad } \\
\text { (promedio })\end{array}$ & 16,5 & 15,3 & 15,6 & 16,6 \\
\hline Región & Huamanga & Lima & Lima & $\begin{array}{l}\text { Ciudad de } \\
\text { México }\end{array}$ \\
\hline
\end{tabular}

Tabla 2 
https://doi.org/10.46744/bapl.201801.005

\subsection{Factores}

Se incluyen dos factores lingüísticos: el tipo de verbo en la cláusula principal y el tipo de evento. Tomando como base la clasificación de Suñer y Padilla-Rivera, este estudio incluye verbos muy estrictos (verbos de deseo) y poco estrictos (verbos factivo-emotivos) en el cumplimiento de CT. Con respecto al segundo factor, se comparan dos tipos de eventos: aquellos que aún son posibles de realizar en el momento del habla (eventos presentes) y aquellos que solo eran posibles de realizar en el pasado (eventos pasados). De acuerdo con lo planteado en el marco teórico, el segundo tipo de evento exige, en términos normativos, que se cumpla con el principio de CT.

\subsection{Instrumento}

Se utilizó un test de producción para medir la frecuencia de presente e imperfecto subjuntivo. Los participantes debían completar oraciones (44 en total) intercaladas con distractores (22 en total). Todas las oraciones incluían uno de los dos tipos de verbo (deseo y factivo-emotivos) y uno de los dos tipos de eventos (presente y pasado). El test presentaba una historia breve que podía ser leída y escuchada. Todas las respuestas fueron grabadas. El ejemplo (7) es uno de los ítems utilizados en el test de producción.

Contexto: Tomás regresa a su casa después del trabajo y encuentra un paquete de libros en la mesa del comedor. Su esposa le cuenta que un vecino los dejó allí en la tarde.

Su esposa dice: «Vino el señor Martínez y preguntó por ti, pensaba que tú ya habías regresado del trabajo. Te trajo esos libros, porque sabe que te gusta la filosofía. El quería que tú [...]».

Algunas respuestas producidas²:

- Pretérito imperfecto subjuntivo: leyeras / estudiaras todos los días / aprendieras más

- Presente subjuntivo: estudies / leas esos libros / estés aquí

2 Las respuestas fueron codificadas identificando el modo gramatical (subjuntivo, indicativo, condicional) y el tiempo (presente o pasado, sobre todo para el subjuntivo). 
- Otros modos gramaticales: trabajabas con la filosofía / leerías los libros

\section{Resultados}

Los resultados generales aparecen en la Tabla 3 organizados según los factores lingüísticos y los grupos de hablantes. Además, las respuestas están divididas según la forma verbal: pasado subjuntivo ${ }^{3}$, presente subjuntivo y otros modos gramaticales.

Tipos de respuestas y porcentajes

\begin{tabular}{|c|c|c|c|}
\hline & $\begin{array}{c}\text { Pasado } \\
\text { subjuntivo }\end{array}$ & $\begin{array}{c}\text { Presente } \\
\text { subjuntivo }\end{array}$ & $\begin{array}{c}\text { Otros modos } \\
\text { gramaticales }\end{array}$ \\
\hline $\begin{array}{c}\text { Bilingüe } \\
(1012 \text { respuestas })\end{array}$ & $\begin{array}{c}437 \\
(43,18 \%)\end{array}$ & $\begin{array}{c}141 \\
(13,93 \%)\end{array}$ & $\begin{array}{c}409 \\
(40,42 \%)\end{array}$ \\
\hline $\begin{array}{c}\text { Más migrante } \\
(1188 \text { respuestas })\end{array}$ & $\begin{array}{c}821 \\
(69,11 \%)\end{array}$ & $\begin{array}{c}180 \\
(15,15 \%)\end{array}$ & $\begin{array}{c}174 \\
(14,65 \%)\end{array}$ \\
\hline $\begin{array}{c}\text { Menos migrante } \\
(1056 \text { respuestas })\end{array}$ & $\begin{array}{c}669 \\
(63,35 \%)\end{array}$ & $\begin{array}{c}181 \\
(17,14 \%)\end{array}$ & $\begin{array}{c}180 \\
(17,05 \%)\end{array}$ \\
\hline $\begin{array}{c}\text { Mexicano } \\
(836 \text { respuestas })\end{array}$ & $\begin{array}{c}689 \\
(82,42 \%)\end{array}$ & $\begin{array}{c}28 \\
(3,35 \%)\end{array}$ & $\begin{array}{c}118 \\
(14,12 \%)\end{array}$ \\
\hline
\end{tabular}

Tabla 3

Como se puede ver en la Tabla 3, las respuestas en pasado subjuntivo, que cumplen con el principio de CT, representan el mayor porcen-

3 Mayoritariamente se refieren al imperfecto, pero también se produjeron respuestas en pretérito perfecto y pluscuamperfecto. Es preciso aclarar, por otro lado, que la Tabla 3 solo presenta las respuestas en que se produjo una forma verbal. Aquellas respuestas agramaticales o que produjeron otras categorías gramaticales fueron descartadas; sin embargo, están incluidas en el número total de respuestas debajo de cada grupo. 
https://doi.org/10.46744/bapl.201801.005

taje de respuestas para todos. Sin embargo, es evidente también que el grupo mexicano es significativamente diferente de los otros tres, ya que estas respuestas son mayoritarias $(82,42 \%)$.

En el caso de los peruanos, incluso el grupo bilingüe tiene mayor porcentaje de pasado subjuntivo, pero con una diferencia muy baja con respecto a otro tipo de respuestas. En cuanto a estas últimas, el presente subjuntivo, vale decir, el no cumplimiento de CT, está presente de manera más o menos uniforme en los grupos peruanos, lo que además representa una amplia ventaja al compararlos con el mexicano. Por otra parte, se observa un porcentaje considerable de respuestas en otros modos gramaticales en todos, pero es el bilingüe el que más llama la atención, pues $40,42 \%$ es un porcentaje muy cercano al de pasado subjuntivo. Estas respuestas se discutirán posteriormente.

El pasado subjuntivo, además, debe evaluarse en términos de los dos factores lingüísticos considerados: tipo de verbo en la cláusula principal y tipo de evento. La Tabla 4 presenta estos resultados; como se puede ver, los porcentajes representan los cuatro contextos usados en las historias del test de producción al tomar en conjunto los dos factores. Así, el primer contexto toma en cuenta los verbos factivo-emotivos y los eventos presentes, lo que constituye el contexto más flexible en cuanto al cumplimiento de CT. En el otro extremo, por el contrario, los verbos de deseo y los eventos pasados constituyen el contexto más estricto en el que la normativa exige CT.

Respuestas en pasado subjuntivo

\begin{tabular}{|c|c|c|c|c|}
\hline & $\begin{array}{l}\text { Factivo- } \\
\text { emotivo } \\
\text { / Eventos } \\
\text { presentes }\end{array}$ & $\begin{array}{c}\text { Deseo / } \\
\text { Eventos } \\
\text { presentes }\end{array}$ & $\begin{array}{l}\text { Factivo- } \\
\text { emotivo } \\
\text { / Eventos } \\
\text { pasados }\end{array}$ & $\begin{array}{l}\text { Deseo / } \\
\text { Eventos } \\
\text { pasados }\end{array}$ \\
\hline
\end{tabular}


Claudia Crespo del Río

https://doi.org/10.46744/bapl.201801.005

\begin{tabular}{|c|c|c|c|c|}
\hline Bilingüe & $37,50 \%$ & $59,78 \%$ & $41,85 \%$ & $58,70 \%$ \\
\hline $\begin{array}{c}\text { Más } \\
\text { migrante }\end{array}$ & $71,83 \%$ & $73,61 \%$ & $75,46 \%$ & $80,09 \%$ \\
\hline $\begin{array}{c}\text { Menos } \\
\text { migrante }\end{array}$ & $56,77 \%$ & $72,92 \%$ & $53,12 \%$ & $78,65 \%$ \\
\hline Mexicano & $65,13 \%$ & $95,39 \%$ & $76,97 \%$ & $97,37 \%$ \\
\hline
\end{tabular}

Tabla 4

Estos resultados fueron analizados estadísticamente usando un ANOVA mixto de $2 \times 2 \times 3$ con los tres factores que aparecen en la tabla (dos lingǘ́sticos y uno social). Los resultados muestran que el tipo de verbo tiene un efecto significativo, $F(1,67)=53.342$, $p<.05$. Si la diferencia es significativa entre los verbos factivo-emotivos y los de deseo, es necesario observar los porcentajes de pasado subjuntivo únicamente con este factor.

Respuestas en pasado subjuntivo con tipo de verbo

\begin{tabular}{|c|c|c|}
\hline & Factivo-emotivo & Deseo \\
\hline Bilingüe & $41,85 \%$ & $58,70 \%$ \\
\hline Más migrante & $73,65 \%$ & $76,85 \%$ \\
\hline Menos migrante & $54,95 \%$ & $75,78 \%$ \\
\hline Mexicano & $71,05 \%$ & $96,38 \%$ \\
\hline Promedio & $60,38 \%$ & $76,93 \%$ \\
\hline
\end{tabular}

Tabla 5 
La Tabla 5 confirma la diferencia significativa entre ambos tipos de verbos al observar los promedios de cada uno: 60,38\% de respuestas en pasado subjuntivo para factivo-emotivos y $76,93 \%$ para deseo. Esta amplia diferencia se puede explicar recordando la clasificación de Suñer y Padilla-Rivera: los verbos de deseo son mucho más estrictos en el cumplimiento de CT. Los factivo-emotivos, más bien, son mucho más flexibles, de ahí que el porcentaje sea más bajo.

El análisis estadístico arrojó una interacción significativa entre el tipo de verbo y el grupo de hablantes, $\mathrm{F}(2,67)=8.840$, $\mathrm{p}<.05$. De este modo, además de que, en promedio, el pasado subjuntivo aparece más con los verbos de deseo, el porcentaje más alto corresponde al grupo mexicano $(96,38 \%)$. Por el contrario, los verbos factivo-emotivos, que son los más flexibles en el cumplimiento de CT, presentan los porcentajes más bajos; dentro de estos, el grupo que menos pasado subjuntivo produjo con este tipo de verbos es el bilingüe $(41,85 \%)$.

Asimismo, el segundo factor lingüístico, el tipo de evento, también tuvo un efecto significativo, $\mathrm{F}(1,67)=5.621, \mathrm{p}<.05$. Por lo tanto, es necesario observar los porcentajes concentrándonos solo en este factor, tal como los presenta la Tabla 6.

Respuestas en pasado subjuntivo por tipo de evento

\begin{tabular}{|c|c|c|}
\hline & Eventos presentes & Eventos pasados \\
\hline Bilingüe & $48,64 \%$ & $50,28 \%$ \\
\hline Más migrante & $72,72 \%$ & $77,77 \%$ \\
\hline Menos migrante & $64,84 \%$ & $65,88 \%$ \\
\hline Mexicano & $80,26 \%$ & $87,17 \%$ \\
\hline Promedio & $66,62 \%$ & $70,28 \%$ \\
\hline
\end{tabular}

Tabla 6 
La Tabla 6 confirma que el pasado subjuntivo aparece más con eventos pasados, es decir, con aquellos que ya no son posibles de realizar en el momento de la comunicación. Estos resultados son acordes con el principio de CT, pues hay mayor flexibilidad de aceptar presente subjuntivo con eventos presentes, o sea, los que aún son posibles de realizar en el momento de la comunicación.

Finalmente, es en relación con el factor grupo que el análisis estadístico mostró un efecto significativo también, $\mathrm{F}(1,67)=1861.649$, $\mathrm{p}<.05$. La Tabla 7, copia parcial de la Tabla 3, nos muestra nuevamente los resultados para pasado subjuntivo en los cuatro grupos.

\section{Respuestas en pasado subjuntivo por grupo}

\begin{tabular}{|c|c|}
\hline Grupo & Pasado subjuntivo \\
\hline Bilingüe (1012 respuestas) & $437(43,18 \%)$ \\
\hline Más migrante (1188 respuestas) & $821(69,11 \%)$ \\
\hline Menos migrante (1056 respuestas) & $669(63,35 \%)$ \\
\hline Mexicano (836 respuestas) & $689(82,42 \%)$ \\
\hline
\end{tabular}

Tabla 7

Los porcentajes entre paréntesis señalan que existe una amplia diferencia entre el grupo bilingüe y el mexicano, que cumplió con el principio de CT en casi $40 \%$ de respuestas más. Esta diferencia resultó estadísticamente significativa $(\mathrm{p}<.05)$ de acuerdo con el test HSD de Tukey de comparaciones pareadas, incluso al comparar los grupos mexicano y menos migrante.

\section{Discusión}

Al enfocarnos primero en los factores lingüísticos, los análisis estadísticos señalaron que, en ambos, hay efectos significativos. Estos se deben a las di- 
https://doi.org/10.46744/bapl.201801.005

ferencias en la producción de pasado subjuntivo entre verbos factivo-emotivos y de deseo, tal como Suñer y Padilla-Rivera describen: los primeros son menos estrictos en el cumplimiento de CT. Por otra parte, con respecto al segundo factor, también se encontraron diferencias significativas entre eventos presentes y pasados, puesto que hubo menor producción de pasado subjuntivo con los primeros. Por consiguiente, la segunda hipótesis de nuestro estudio puede ser confirmada: el cumplimiento de CT es mayor con el tipo de verbo y el tipo de evento más estrictos.

Los factores lingüísticos, entonces, reafirman los hallazgos encontrados en estudios previos; lo que resulta más llamativo en el nuestro, por tanto, es el factor social. Los análisis estadísticos y las comparaciones subrayan las grandes diferencias en el cumplimiento de CT por parte de los grupos. Por ejemplo, notamos que, de las cuatro, es la variedad mexicana la que efectivamente sirve de grupo de control; en otras palabras, es la que sigue el patrón normativo del principio de CT en la gran mayoría de casos. El porcentaje y el análisis estadístico permiten confirmar la primera hipótesis: las variedades de castellano peruano se alejan más del principio de CT que las mexicanas.

Por otro lado, el análisis estadístico también señaló que el grupo bilingüe se diferenciaba claramente de los otros tres, ya que es el que menos respuestas en pasado subjuntivo produjo. Este resultado sugiere que revisemos si la tercera hipótesis se confirma: las variedades con menor contacto migrante cumplirán con el principio de CT en mayor medida que las variedades con mayor contacto migrante. Tal hipótesis se desglosaba de la siguiente manera tomando en cuenta nuestros grupos peruanos: grupo menos migrante $>$ grupo más migrante $>$ grupo bilingüe.

Con respecto al grupo bilingüe, el porcentaje bajo de respuestas en pasado nos señala que el principio de CT no se cumple tanto como en los otros dos grupos; tal hallazgo sugiere que la hipótesis se puede confirmar parcialmente (el grupo bilingüe se ubica en el extremo de menor uso de pasado subjuntivo). No obstante, al fijarnos en los dos grupos limeños, 
encontramos que la diferencia no es amplia ni estadísticamente significativa $(69,11 \%$ vs. $63,35 \%)$. De esta manera, es posible entender que la tercera hipótesis no se cumple en su totalidad, puesto que el grupo más migrante tiene un porcentaje mayor al del menos migrante; o sea, el primero se acerca más a la variedad normativa.

Para explicar por qué el resultado es inverso al esperado, vale la pena considerar las características sociales y lingüísticas de ambos grupos. Se trata de hablantes que viven en la misma ciudad, y cuyos intereses y pasatiempos son similares debido a su rango de edad (ir al cine, salir con amigos, practicar deportes, estudiar idiomas, etc.); estos datos fueron obtenidos a partir del cuestionario sociolingüístico aplicado a cada entrevistado.

Por otra parte, el principal criterio de diferenciación entre ambos grupos es el contacto con migrantes, determinado por el área o distrito de residencia de los hablantes. Si bien los datos demográficos y otras características sociales nos muestran que la concentración de migrantes andinos en los dos distritos estudiados es distinta (Arellano y Burgos 2010), esto no descarta completamente que los participantes tengan contacto con otros grupos a través de las actividades que realizan y de los medios de comunicación. Por este motivo, se puede entender que el cumplimiento no estricto de CT sea parecido para ambas variedades, sobre todo debido a que no se trata de un fenómeno estigmatizado o identificado con alguna variedad en particular.

Por lo tanto, la tercera hipótesis no se confirma completamente. Solo puede comprobarse que el grupo bilingüe de la región andina es el que menos cumple con el principio de CT. Sin embargo, es necesario explicar el porqué de este resultado, ya que, al contrario de lo que se podría suponer, el menor porcentaje de pasado subjuntivo en este grupo no se debe a la preferencia por presente subjuntivo, tal como se observa en el español peruano en general en otros estudios ya revisados. De acuerdo con la Tabla 3 ya presentada, los bilingües produjeron $13,93 \%$ de sus respuestas en esa forma verbal. Son, más bien, las respuestas en otros modos gramaticales las que representan un porcen- 
https://doi.org/10.46744/bapl.201801.005

taje similar al pasado subjuntivo: 40,42\%. Ninguno de los otros grupos produjo un porcentaje tan alto de otro tipo de respuestas. Los bilingües, entonces, eligieron completar muchas de las oraciones con verbos en indicativo o en condicional ${ }^{4}$.

Las respuestas nos muestran que estas construcciones no son comúnmente elegidas por un hablante monolingüe de castellano, incluso con verbos en la cláusula principal que exigen el uso del subjuntivo (como los de deseo). Por ello, prestamos atención al hecho de que los miembros del grupo ayacuchano sean todos hablantes de quechua.

Los autores interesados en el español en contacto con el quechua afirman que existe una preferencia por el indicativo en contextos de subjuntivo (Calvo Pérez 2009, Caravedo 1992, Carranza Romero 1993, Escobar 1978, Escobar 2000). Además, una tendencia como esta es aun mayor en hablantes cuyo dominio del castellano es menor. Así, resulta necesario observar si todos los participantes bilingües comparten un nivel de dominio cercano, lo que fue evaluado a través de un test de español como segunda lengua de 40 preguntas (usado en Santos, 2013). En este, los hablantes debían elegir la opción correcta entre cuatro alternativas para completar, por un lado, un texto y, por otro lado, una serie de oraciones. El dominio de los hablantes es medido en términos de respuestas correctas: más de 30 corresponden a un nivel más dominante y de 21 a 30, a un nivel menos dominante.

Los resultados del test dividieron al grupo bilingüe: 17 de los 23 participantes alcanzaron el nivel más dominante de español, lo cual demuestra que, en efecto, la mayoría de ellos reportaron hablar español desde su infancia temprana, pues adquirieron el quechua y el castellano simultáneamente. Por otra parte, los seis restantes alcanzaron un nivel menos dominante, lo que coincide con los participantes que reportaron haber aprendido el español como segunda lengua, es

4 Ejemplos reales extraídos del grupo bilingüe fueron presentados en la sección 3.3 (Instrumento). 
decir, después de haber adquirido el quechua como lengua materna. La diferencia de puntajes entre ambos subgrupos es estadísticamente significativa según el ANOVA unifactorial realizado, $\mathrm{F}(1,22)=$ 45.879, $\mathrm{p}<.05$. Los tipos de respuestas para completar el test de producción son distintos al separar el grupo bilingüe en dos, tal como lo muestra la Tabla 8.

Respuestas de los subgrupos bilingües

\begin{tabular}{|c|c|c|c|c|}
\hline & $\begin{array}{c}\text { Pasado } \\
\text { subjuntivo }\end{array}$ & $\begin{array}{c}\text { Presente } \\
\text { subjuntivo }\end{array}$ & Indicativo & Condicional \\
\hline $\begin{array}{c}\text { Subgrupo } \\
\text { más } \\
\text { dominante }\end{array}$ & $46,12 \%$ & $15,51 \%$ & $31,28 \%$ & $5,88 \%$ \\
\hline $\begin{array}{c}\text { Subgrupo } \\
\text { menos } \\
\text { dominante }\end{array}$ & $34,85 \%$ & $9,47 \%$ & $37,5 \%$ & $12,12 \%$ \\
\hline
\end{tabular}

Tabla 8

$\mathrm{Al}$ enfocarnos en las respuestas que cumplen con el principio de $\mathrm{CT}$, resulta notorio que el subgrupo más dominante es el que más respuestas de este tipo produjo (más de $11 \%$ de diferencia) y también más respuestas en presente subjuntivo. Esto refleja un comportamiento más parecido al de los grupos monolingües, lo que fue confirmado estadísticamente a través de un test de chi-cuadrado, elegido por ser no paramétrico, ideal para comparar grupos que tienen un número de miembros muy diferente. El test mostró una asociación significativa entre el nivel de dominio de castellano y el tipo de respuesta, X2(1) $=5.933, \mathrm{p}<.05$ (pasado subjuntivo) y X2(1) $=10.110, \mathrm{p}<.05$ (presente subjuntivo).

Entonces, el subgrupo menos dominante produjo respuestas más alejadas de los grupos monolingües y abre la pregunta de por qué tienen preferencia por otros modos gramaticales. La respuesta se halla en los 
https://doi.org/10.46744/bapl.201801.005

estudios de adquisición de segundas lenguas: el subjuntivo no es un rasgo que se desarrolle en etapas tempranas del proceso de adquisición (Geeslin y Gudmestad 2008, Gudmestad 2012, Lubbers Quesada 1998); pues adquiere rasgos léxicos y semánticos completamente en los niveles más avanzados. Esos rasgos, que son los que guían a los hablantes nativos, se vuelven realmente relevantes para los aprendices tardíamente (Collentine 2010, Gudmestad 2012).

\section{Conclusiones}

De las tres hipótesis planteadas en el estudio, es posible confirmar la primera y la segunda de manera contundente. Por un lado, los datos aseguran que el español peruano (o las variedades que lo conforman) no sigue estrictamente el principio de CT o que, por lo menos, se aleja más que otros dialectos latinoamericanos, como el mexicano. Esta confirmación deja abierta la discusión sobre si se trata de un cambio lingüístico en progreso, lo que llevaría a que las variedades peruanas se encuentren un paso más adelante en el uso de formas verbales de subjuntivo. Otras lenguas romances, como el francés, evidencian que este modo gramatical es objeto de cambio permanente, por lo que se predice que un fenómeno similar pueda suceder en el castellano.

Si un cambio está en progreso en el español peruano, es relevante que la hipótesis acerca de los factores lingüísticos haya sido confirmada. Aquellos contextos lingüísticos que son más estrictos en exigir el cumplimiento del principio de CT son los que mayores frecuencias de pasado subjuntivo produjeron. Esto se confirma tanto para verbos de deseo como para eventos pasados; así, se puede suponer que estos contextos estrictos serán los últimos en los que el cambio a presente subjuntivo aparezca.

Por último, la tercera hipótesis del estudio fue confirmada parcialmente, debido a que las dos variedades habladas en Lima no presentaron diferencias significativas. Nuestra propuesta es que, como se señaló al inicio de este artículo, al tratarse de grupos residentes en la capital que comparten muchas características sociales, existen muchas coincidencias 
e intenso contacto entre migrantes y no migrantes en general. Por esta razón, ambos grupos se comportan de manera similar.

No obstante, la última hipótesis se confirma del lado del grupo bilingüe, ya que este fue el que menos cumplió con el principio de CT. Lo más interesante de este grupo, sin embargo, es que sus bajos porcentajes de pasado subjuntivo no se deben a una preferencia por el presente subjuntivo, sino por otros modos gramaticales, como el indicativo. Estudios en adquisición de segundas lenguas sugieren que este resultado inesperado se explica por el aprendizaje tardío del subjuntivo, lo que cobra mayor sustento al dividir el grupo bilingüe en dos subgrupos según su nivel de dominio del castellano: quienes tienen un menor dominio son quienes más respuestas en otros modos gramaticales produjeron.

Para terminar, es necesario sugerir que el estudio del principio de CT en variedades peruanas sea ampliado a otro tipo de cláusulas subordinadas para confirmar si es realmente un cambio en progreso que se está expandiendo. También, se debe considerar la influencia de otros factores tanto lingüísticos como sociales para elaborar pronósticos acerca del futuro del subjuntivo en estas variedades. 
https://doi.org/10.46744/bapl.201801.005

\section{BIBLIOGRAFÍA}

ARELLANO, R. y BURGOS, D. (2010). Ciudad de los Reyes, de los Chávez, de los Quispe... Lima: Planeta.

ARRIZABALAGA, C. (2009). Imploraba que no lo maten. Reorganización de los tiempos del subjuntivo en español peruano. Moenia, $15,295-311$.

CALVO, J. (2009). Persiguiendo una necesidad: influencia de las lenguas andinas en el español del Perú. En Calvo, J. y Miranda, L. (Eds.), Palabras fuera del nido. Vertientes sincrónica y diacrónica del español en contacto. Lima: Universidad de San Martín de Porres, 89-108.

CARAVEDO, R. (1992). Espacio geográfico y modalidades lingüísticas en el español del Perú. En HERNÁNDEZ, C. (Coord.), Historia y presente del español de América. Junta de Castilla y León: Pabecal, 719-742.

CARAVEDO, R. y KLEE, C. (2005). Contact-induced language change in Lima, Peru: the case of clitic pronouns. En EDDINGTON, D. (Ed.), Selected Proceedings of the 7th Hispanic Linguistics Symposium. Somerville, MA: Cascadilla Proceedings Project, 12-21.

CARRANZA, F. (1993). Resultados lingüísticos del contacto quechua y español. Trujillo: Libertad.

CERróN-PALOMINO, R. (2003). Castellano andino. Lima: Pontificia Universidad Católica del Perú.

COLlENTINE, J. (2010). The Acquisition and Teaching of the Spanish Subjunctive: An Update on Current Findings. Hispania, 93(1), 39-51. 
https://doi.org/10.46744/bapl.201801.005

DE GRANDA, G. (2003). Estudios lingüisticos hispanoamericanos. Historia, sociedades y contactos. New York: Lang.

ESCOBAR, A. (1978). Variaciones sociolingüísticas del castellano en el Perú. Lima: Instituto de Estudios Peruanos.

ESCOBAR, A. M. (2000). Contacto social y lingüústico: el español en contacto con el quechua en el Perú. Lima: Pontificia Universidad Católica del Perú.

. (2001). La Relación de Pachacuti, ¿español andino o español bilingüe? Lexis, 25(1-2), 115-136.

. (2007). Reflexiones sobre el cambio semántico: el caso de de que en el español andino. Signo y Seña, 18, 57-71.

. (2009). La gramaticalización de estar+gerundio y el contacto de lenguas. En ESCOBAR, A. M. y WÖLCK, W. (Eds.), Contacto lingüístico y la emergencia de variantes $y$ variedades lingüísticas. Madrid/Frankfurt: Iberoamericana/ Vervuert, 39-63.

GEESLIN, K. y GUDMESTAD, A. (2008). Comparing Interview and Written Elicitation Tasks in Native and Non-native Data: Do Speakers Do What We Think They Do? En BRUHN DE GARAVITO, J. y VALENZUELA, E. (Eds.), Selected Proceedings of the 10th Hispanic Linguistics Symposium. Somerville, MA: Cascadilla Proceedings Project, 64-77.

GILI GAYA, S. (1948). Curso superior de sintaxis española. Barcelona: Publicaciones y Ediciones Spes.

GUDMESTAD, A. (2012). Acquiring a Variable Structure: An Interlanguage Analysis of Second Language Mood Use in Spanish. Language Learning, 62(2), 373-402. 
https://doi.org/10.46744/bapl.201801.005

LUBBERS QUESADA, M. (1998). L2 Acquisition of the Spanish Subjunctive Mood and Prototype Schema Development. Spanish Applied Linguistics, 2(1), 1-23.

MICK, C. y PALACIOS, A. (2013). Mantenimiento o sustitución de rasgos lingüísticos indexados socialmente: migrantes de zonas andinas en Lima. Lexis, XXXVII (2), 341-380.

PÉREZ, J. I. (2004). Los castellanos del Perú. Lima: PROEDUCA - GTZ.

SANTOS, H. (2013). Cross-linguistic influence in the acquisition of Brazilian Portuguese as a third language. Tesis para optar el grado de doctora en Lingüística Hispánica. Urbana, University of Illinois at Urbana-Champaign.

SESSAREGO, S. (2008). Spanish Concordantia Temporum: an old issue, new solutions. En WESTMORELAND, M. y THOMAS, J.A. (Eds.), Selected proceedings of the 4th workshop on Spanish sociolinguistics. Somerville, MA: Cascadilla Proceedings Project, 91-99.

. (2010). Temporal concord and Latin American Spanish dialects: a genetic Blueprint. Revista Iberoamericana de Lingüística, 5, 137-169.

SUÑER, M. y PADILLA-RIVERA, J. (1987). Sequence of tenses and the subjunctive. Hispania, 70(3), 634-642.

ZAVALA, V. (1999). Reconsideraciones en torno al español andino. Lexis, XXIII (1), 25-86. 